GEOMETRY IN NONLINEAR CONTROL

AND DIFFERENTIAL INCLUSIONS

BANACH CENTER PUBLICATIONS, VOLUME 32

INSTITUTE OF MATHEMATICS

POLISH ACADEMY OF SCIENCES

WARSZAWA 1995

\title{
CONTROLLABILITY OF RIGHT INVARIANT SYSTEMS ON SEMI-SIMPLE LIE GROUPS
}

\author{
R. EL ASSOUDI \\ INSA de Rouen, LMI, AMS, URA CNRS 1378 \\ BP 08, Place Emile Blondel, 76131 Mont St Aignan, France \\ J. P. GAUTHIER \\ INSA de Rouen, LMI, AMS, URA CNRS 1378 \\ BP 08, Place Emile Blondel, 76131 Mont St Aignan, France \\ Institut Universitaire de France \\ I. A. K. KUPKA \\ Department of Mathematics, University of Toronto \\ 100 St Georges Street, Toronto, Ontario, M5S-1A1, Canada
}

\begin{abstract}
We deal with controllability of right invariant control systems on semi-simple Lie groups. We recall the history of the problem and the successive results. We state the final complete result, with a sketch of proof.

0. Introduction, History of the problem, previous results. In this paper, $\mathbb{R}$ and $\mathbb{C}$ denote respectively the fields of real and complex numbers. For the statement of the problem, we refer to the notations defined in the next section 1. We deal with the transitivity (or controllability) of families of right (or left) invariant vector fields $\Gamma$ on a real semi-simple Lie group $G$, with finite center whose Lie algebra is denoted by $L$. Let $\Gamma \subset L, \Gamma$ is said to be transitive if the sub-semigroup $\Gamma^{+}$of $G$ generated by $\{\exp t X \mid X \in \Gamma, t \geq 0\}$ is all of $G$.

This question of transitivity is the basic problem of control theory. It is especially important to solve the case of right invariant systems on semi-simple Lie

1991 Mathematics Subject Classification: Primary 93B05, 17B20; Secondary 93C10, 22E46.

Key words and phrases: semi-simple Lie algebras, controllability, invariant vector fields, root systems.

Research of the second and third authors partly supported by NSERC GRANT OGP 0036498.

The paper is in final form and no version of it will be published elsewhere.
\end{abstract}


groups for several reasons. One of these reasons is that it allows to produce controllability theorems in more "practical" cases: certain semi-direct product groups and the so called bilinear "systems" on $\mathbb{R}^{n}$ (see [BJKS] for an example).

Controllability on other types of Lie groups has been dealt with by several authors: see for instance [HHL], [L], [HI].

Affine families of vector-fields, i.e. $\Gamma=\{A+u B \mid u \in \mathbb{R},(A, B) \in L \times L\}$ are especially important in control theory, since control systems often appear naturally under the form $\dot{x}=(A+u B) x$. The techniques that we deal with are particularly efficient in this case. Our purpose in this paper is, after having recalled the history of the problem and the successive results, to state a final version of the theorem (theorem 2 below) and to give a sketch of its proof.

All papers dealing with controllability on semi-simple Lie groups make use of the extension techniques proposed in the original paper $[\mathrm{JK}]$. In this paper, the first theorem on simple Lie groups is stated:

Theorem 0 ([JK], Theorem 0, page 156). Let $G$ be a real connected Lie group with finite center whose Lie algebra $L$ is a real form of a simple Lie algebra $L_{\mathbb{C}}$. $A$ subset $\Gamma \subset L$ is transitive if:

1) $\Gamma$ contains a one dimensional space $\mathbb{R} B$ where $B$ is strongly regular,

2) $\Gamma$ satisfies the "strong rank condition" with respect to $B$,

3) let $s=\sup \{\operatorname{Re} a \mid a \in \operatorname{Sp} B\}$. In the case where $s \notin \operatorname{Sp} B$, there are $a_{1}, a_{2} \in \operatorname{Sp} B$ and $A_{1}, A_{2} \in \Gamma$ such that $\operatorname{Re} a_{1}=s=-\operatorname{Re} a_{2}$ and $A_{1}\left(a_{1}\right) \neq 0$, $A_{2}\left(a_{2}\right) \neq 0$,

4) in the case where $s \in \operatorname{Sp} B$, there are $A_{1}, A_{2} \in \Gamma$ such that $\operatorname{Trace}\left(\operatorname{ad} A_{1}(s) \circ\right.$ ad $\left.A_{2}(-s)\right)<0$.

See $[\mathrm{JK}]$ for the "strong rank condition". There is also theorem 1, page 157 of the same paper, dealing with the semi-simple case.

In previous papers we have already improved these results in several cases by showing that assumption 2 (strong rank condition) can be replaced by the weaker, more natural (and obviously necessary for controllability) simple rank condition, i.e. the Lie algebra generated by $\Gamma, \operatorname{Lie}(\Gamma)$, is equal to $L$.

1) First, in the paper [GB] this criterion was proved to be sufficient for $G=$ $\operatorname{SL}(n, \mathbb{R})$ with ad $B$ real diagonalizable (theorem 2 , page 378 ).

2) Second, the same result was generalized in [GKS] for some classes of simple Lie groups: when $L$ is a real normal - or split - form of any complex Lie algebra of type $A_{n}, D_{n}, E_{6}, E_{7}, E_{8}$ with the same assumption that $B$ is real strongly regular, i.e. ad $B$ is real diagonalizable ([GKS], theorem 1, page 188).

3) Third, the same result was proved to be true for other remaining types $G_{2}$, $F_{4}, B_{n}, C_{n}$ in [EAG] (theorem 2, page 295):

THEOREM 1 [EAG]. Let $G$ be a real connected Lie group with finite center whose Lie algebra $L$ is a real normal form of a simple Lie algebra $L_{\mathbb{C}}$. Assume that $\Gamma=\{A+u B \mid u \in \mathbb{R},(A, B) \in L \times L\}$, satisfies the following conditions. 
1) $B$ is "real strongly regular".

2) If $A=A_{0}+\sum_{a \in \mathrm{Sp} B} A_{a}$, and $a$ is maximal with respect to $B$, then

$$
\text { Trace }\left(\operatorname{ad} A_{+a} \circ \text { ad } A_{-a}\right)<0 .
$$

In the paper [LC] (theorem 5, page 40) the same result is proved for $A_{n}, D_{n}$ with a slightly weaker assumption on $B$ (i.e. $B$ is only " $A$-real strongly regular", see $[\mathrm{LC}]$ for this assumption).

Now, let us state the final result, which is the main result of this paper:

THEOREM 2. Let $G$ be a real connected semi-simple Lie group with finite center and let $L$ be its Lie algebra. Let $\Gamma=\{A+u B \mid u \in \mathbb{R}\},(A, B) \in L \times L$, satisfy the following conditions.

(H1) B is "strongly regular".

(H2) $\Gamma$ satisfies the rank condition, i.e. $\operatorname{Lie}(\Gamma)=L$.

(H3) Let $A=A_{0}+\sum_{a \in \mathrm{Sp} B} A_{a}$. If a is maximal or minimal with respect to $B$, then $A_{a} \neq 0$.

(H4) If $a$ and $\alpha \in \operatorname{Sp} B$, a is maximal and $\alpha=\operatorname{Re} a, L_{\alpha}$ and $L_{a}$ being in the same simple component of $L_{\mathbb{C}}$, then $\operatorname{Trace}\left(\operatorname{ad} A_{\alpha} \circ\right.$ ad $\left.A_{-\alpha}\right)<0$.

Then $\Gamma$ is transitive.

In this paper, we give a sketch of the proof of this final result (Theorem 2 above). This proof is somewhat different from the basic one in [JK]. It is based upon:

1. Elementary properties of the Lie saturated set $\operatorname{LS}(\Gamma)$ associated to a subset $\Gamma$ of the Lie algebra $L$ of $G$ (see section 2).

2. Properties of the root systems of simple Lie algebras (see proposition 0 , section 1).

The previous properties, together with the extension techniques proposed in the paper [JK], are used in the last section to prove two key results (lemmas 3, 4). These lemmas are important to produce a new result (theorem 3), which, in turn, implies obviously the main theorem.

1. Basic definitions and results. Let $L$ be a real semi-simple Lie algebra and $L_{\mathbb{C}}=L \otimes_{\mathbb{R}} \mathbb{C}$ be its complexification. Let $\sigma_{L}: L_{\mathbb{C}} \rightarrow L_{\mathbb{C}}$ be the conjugation associated to $L$.

A "strongly regular" element $B$ is an element of $L$ such that $\operatorname{ad}_{\mathbb{C}} B$, the complexification of $\operatorname{ad} B$, is diagonalizable, $\operatorname{ker}_{\mathbb{C}} B$ is a Cartan subalgebra of $L_{\mathbb{C}}$ and all the nonzero eigenspaces of $\operatorname{ad}_{\mathbb{C}} B$ are one dimensional. Let $\operatorname{Sp} B$ denote the (possibly complex) nonzero spectrum of $\operatorname{ad}_{\mathbb{C}} B$. If $\operatorname{Sp} B \subset \mathbb{R}, B$ is said to be "real strongly regular". (Recall, for the purpose of understanding theorem 1, that a real normal form of a simple Lie algebra contains real strongly regular elements). If $a \in \operatorname{Sp} B \cup\{0\}, L_{a}$ denotes the complex eigenspace of $\operatorname{ad}_{\mathbb{C}} B$, $L_{a}=\operatorname{Ker}\left(\operatorname{ad}_{\mathbb{C}} B-a \mathrm{Id}\right)$ and $L(a)$ denotes the real eigenspace, $L(a)=\left(L_{a}+L_{a^{*}}\right) \cap L$ 
$\left(a^{*}=\right.$ conjugate of $\left.a\right) . A_{a}$ denotes the complex $a$-component of $A$ and $A(a)$ the real $a$-component. Then $A=A_{0}+\sum_{a \in \mathrm{Sp} B} A_{a}$.

The set $\operatorname{Sp} B$ is totally ordered by the lexicographic order over $\mathbb{C}$.

Definition 0. We say that $a \in \operatorname{Sp} B, a>0$ (resp. $a<0$ ), is maximal (resp. minimal) with respect to $B$ if for all $b \in \operatorname{Sp} B, b>0$ (resp. $b<0$ ), $\left[L_{a}, L_{b}\right]=\{0\}$.

Let $L_{\mathbb{C}}=\bigoplus_{i=1}^{k} S_{i}$ be the decomposition into simple components, and let $\eta \subset L_{\mathbb{C}}$ be a Cartan subalgebra invariant by $\sigma_{L}$. We have $\eta=\bigoplus_{i=1}^{k} \eta_{i}$, where $\eta_{i}=$ $\eta \cap S_{i}$ is a Cartan subalgebra of $S_{i}$. We denote by $R$ a root system of $L_{\mathbb{C}}$ relative to $\eta_{i}$ and by $R_{i}$ a root system of $S_{i}$ relative to $\eta_{i}$ then $R \subset \eta^{\prime}=\operatorname{Hom}_{\mathbb{C}}(\eta, \mathbb{C})$ and $R_{i} \subset \eta_{i}^{\prime}=\operatorname{Hom}_{\mathbb{C}}\left(\eta_{i}, \mathbb{C}\right)$. Each $h \in \eta_{i}^{\prime}$ can be canonically extended to $\eta$ as $\eta^{\prime}=\bigoplus_{i=1}^{k} \eta_{i}^{\prime}$ and $R$ is the union of $R_{i}, i=1,2, \ldots, k$.

$\sigma_{L}$ induces an involution $\sigma: \eta^{\prime} \rightarrow \eta^{\prime}$ defined by $\sigma(h)=* \circ h \circ \sigma_{L}$ where $*: \mathbb{C} \rightarrow \mathbb{C}$ is the standard conjugation.

Then $R$ and the $R_{i}, 1 \leq i \leq k$, are stable under the map $\alpha \rightarrow-\alpha$, and $R$ is stable under $\sigma$. Moreover if $\alpha \in R$, then $n \alpha \notin R$ for $n \in \mathbb{R} \backslash\{1,-1\}$. We denote $\operatorname{Re} \alpha=(\alpha+\sigma(\alpha)) / 2$, and $\operatorname{Im} \alpha=\sqrt{-1}(\sigma(\alpha)-\alpha) / 2$.

A "strongly regular" element $B$ can also be defined as follows. Let $B \in L \cap \eta$ be such that the map: $R \cup\{0\} \rightarrow \mathbb{C}, 0 \rightarrow 0, R \ni \alpha \rightarrow \alpha(B)$ is a bijection. $B$ defines an order structure on $\eta^{\prime}$ as follows: $h_{1}>h_{2}$ if $\operatorname{Re} h_{1}(B)>\operatorname{Re} h_{2}(B)$ or if $\operatorname{Re} h_{1}(B)=\operatorname{Re} h_{2}(B)$ and $\operatorname{Im} h_{1}(B)>\operatorname{Im} h_{2}(B)$.

This order structure is compatible with the real vector-space structure of $\eta^{\prime}$. (Caution: $\geq 0$ and $\leq 0$ do not imply $=0$ ). On $R, B$ induces a total order (for any $\alpha, \beta \in R, \alpha>\beta, \alpha<\beta$ or $\alpha=\beta$ ) having the following property: if $\alpha \in R$ and $\operatorname{Re} \alpha \neq 0($ resp. $\operatorname{Im} \alpha \neq 0)$, then $\operatorname{Re} \alpha(B) \neq 0($ resp. $\operatorname{Im} \alpha(B) \neq 0)$.

Equivalently with definition 0 , a root $\alpha \in R$ is said to be maximal (resp. minimal) if $\alpha+\beta \notin R$ for all $\beta \in R$ and $\beta>0$ (resp. $<0$ ).

If $R$ is irreducible, $R$ contains only one maximal root $s$ and one minimal root $-s$. Since the $S_{i}, i=1,2, \ldots, k$, are simple, each $R_{i}$ is irreducible and contains only one maximal root $s_{i}$.

For all $\alpha \in R$, we denote also by $L_{\alpha}$ the $\alpha$-space of $L_{\mathbb{C}}: L_{\alpha}=\left\{X \in L_{\mathbb{C}}\right.$ : $\forall H \in \eta,[H, X]=\alpha(H) X\} . L_{\alpha}$ here coincides with $L_{\alpha(B)}$ defined earlier. The following results are well known (see for instance $[\mathrm{B}],[\mathrm{H}],[\mathrm{W}]$ ):

Lemma 0. a) $\operatorname{dim}_{\mathbb{C}} L_{\alpha}=1$ for all $\alpha \in R$.

b) $L_{\mathbb{C}}=\eta \oplus \bigoplus_{\alpha \in R} L_{\alpha}$.

c) If $\alpha, \beta \in R,\left[L_{\alpha}, L_{\beta}\right]=\{0\}$ if $a+b \notin R$ and $\alpha+\beta \neq 0,\left[L_{\alpha}, L_{\beta}\right]=L_{\alpha+\beta}$ if $\alpha+\beta \in R$, and $\left[L_{\alpha}, L_{\beta}\right]$ has dimension 1 and is contained in $\eta$ if $\alpha+\beta=0$. There are positive integers $p(\alpha, \beta)$ and $q(\alpha, \beta)$ such that $\alpha+m \beta \in R \Leftrightarrow-p(\alpha, \beta) \leq m \leq$ $q(\alpha, \beta)$, where $m$ is an integer.

d) $\sigma_{L}\left(L_{\alpha}\right)=L_{\sigma(\alpha)}$ for all $\alpha \in R$. Hence $L_{\alpha}+L_{\sigma(\alpha)}$ is invariant by $\sigma_{L}$. Define $L(\alpha)=L \cap\left(L_{\alpha}+L_{\sigma(\alpha)}\right)$. Hence $L(\sigma(\alpha))=L(\alpha), L(\alpha) \oplus_{\mathbb{R}} \mathbb{C}=L_{\alpha}+L_{\sigma(\alpha)}$ and $\operatorname{dim}_{\mathbb{R}} L(\alpha)=1$ if $\alpha=\sigma(\alpha), \operatorname{dim}_{\mathbb{R}} L(\alpha)=2$ otherwise. 
e) $L=\eta \cap L \oplus \bigoplus\{L(\alpha) \mid \alpha \in R, \operatorname{Im} \alpha(B) \geq 0\}$.

f) For all $\alpha, \beta \in R,[L(\alpha), L(\beta)]=L(\alpha+\beta)+L(\alpha+\sigma(\beta))$ if $\alpha \neq \sigma(\alpha)$ and $\beta \neq \sigma(\beta)$ or else $[L(\alpha), L(\beta)]=L(\alpha+\beta)$ if $\alpha=\sigma(\alpha)$ or $\beta=\sigma(\beta)$.

Clearly, if $X \in L$, by b), e) above, $X$ has unique decompositions: $X=X_{0}+$ $\sum\left\{X_{\alpha} \mid \alpha \in R\right\}$ and $X=X(0)+\sum\{X(\alpha) \mid \alpha \in R, \operatorname{Im} \alpha(B) \geq 0\}$, where $X_{0}=X(0) \in \eta, X_{\alpha} \in L_{\alpha}, X(\alpha) \in L(\alpha)$ and we have $X(\alpha)=X_{\alpha}$ if $\alpha=\sigma(\alpha)$; $X(a)=X_{\alpha}+\sigma_{L} X_{\alpha}=X_{\alpha}+X_{\sigma(\alpha)}$ if $\alpha \neq \sigma(\alpha)$.

Convention. If $h \in \eta^{\prime}, h \notin R \cup\{0\}$, we set $L_{h}=L(h)=\{0\}$.

If $h=0, L(0)$ denotes a subspace of $\eta \cap L$ (depending on the situation) and $L_{0}$ denotes a subspace of $\eta$. Always, $L(\sigma(h))=L(h)$.

Moreover, if $\alpha \in R$ and $\operatorname{Im} \alpha(B)<0$ we set $X(\alpha)=X(\sigma(\alpha))$. If $h \in \eta^{\prime}$, $h \notin R \cup\{0\}, X(h)=0$. If $h=0, X(0)$ denotes an element of $\eta \cap L$. Always, $X(h)=X(\sigma(h))$.

Lemma 1. Let $X \in L(\alpha) \backslash\{0\}$ and $Y \in L$. Let $B$ be "strongly regular". For any $\beta \in R$ such that $Y(\beta) \neq 0$ and $\alpha+\beta \in R \cup\{0\}$, we have: If $\alpha=\sigma(\alpha)$ or if $Y(\alpha-\sigma(a)+\beta)=0$ then $[X, Y](\alpha+\beta) \neq 0$. If $\alpha \neq \sigma(\alpha)$ and $Y(\alpha-\sigma(\alpha)+\beta) \neq 0$, the set of $t \in \mathbb{R}$ such that $\left[e^{t \text { ad } B}(X), Y\right](\alpha+\beta) \neq 0\left(\right.$ resp. $\left[X, e^{t \text { ad } B}(Y)\right](\alpha+\beta) \neq$ 0 ) is the complement of a discrete subset of $\mathbb{R}$ (possibly empty).

The proof is elementary. We leave it to the reader.

Proposition 0. Let $\operatorname{Max}(R)$ be the set of maximal roots of $R, R^{\prime}$ the subset of roots $\alpha$ such that there exists an $s \in \operatorname{Max}(R)$ for which $\alpha+s$ or $\alpha-s$ is also a root. Set $R^{\prime \prime}=R \backslash\left(R^{\prime} \cup \pm \operatorname{Max}(R)\right)$. Then:

1) If $\alpha, \beta \in R^{\prime}, \alpha, \beta$ have the same sign and $\alpha+\beta \in R$, then $\alpha+\beta \in \pm \operatorname{Max}(R)$.

2) If $\alpha \in R^{\prime}, \beta \in R^{\prime \prime}$ and $\alpha+\beta \in R$, then $\alpha+\beta \in R^{\prime}$ and $\alpha, \alpha+\beta$ have the same sign.

3) If $\alpha, \beta \in R^{\prime \prime}$ and $\alpha+\beta \in R$, then $\alpha+\beta \in R^{\prime \prime}$.

The proof (elementary) can be found in $[\mathrm{J}]$. See also [EA].

Remark 0 . The sets $R^{\prime}$ and $R^{\prime \prime}$ are stable under the map $\alpha \rightarrow-\alpha$. They are not conjugation-stable in general.

Corollary 0. (i) Assume that $\alpha, \gamma \in R^{\prime}, \beta \in R^{\prime \prime}, \alpha, \gamma$ have the same sign and $\alpha+\beta \in R, \alpha+\beta+\gamma \in R$. Then $\alpha+\beta+\gamma$ is maximal or minimal.

(ii) Assume that $\alpha \in R^{\prime}, \beta, \gamma \in R^{\prime \prime}, \alpha+\beta+\gamma \in R$ and moreover that at least one of the two linear forms $\alpha+\beta$ or $\beta+\gamma$ is a root. Then $\alpha+\beta+\gamma \in R^{\prime}$ and $\alpha, \alpha+\beta+\gamma$ have the same sign.

2. Properties of the Lie saturated set $\operatorname{LS}(\Gamma)$. Recall that for $\Gamma \subset L$, $\operatorname{LS}(\Gamma)$ is the largest subset of $\operatorname{Lie}(\Gamma)$ such that closure $\left(\operatorname{LS}(\Gamma)^{+}\right)=\operatorname{closure}\left(\Gamma^{+}\right)$. A necessary and sufficient condition for controllability of $\Gamma$ is just $\operatorname{LS}(\Gamma)=L$ (see $[\mathrm{JK}])$. 
In the rest of the paper, we will consider special subsets $\Gamma$ of $L$, with the following properties:

a) $\operatorname{LS}(\Gamma)$ is a closed convex cone.

b) If $\pm X \in \operatorname{LS}(\Gamma)$ and $Y \in \operatorname{LS}(\Gamma)$, then $e^{t \operatorname{ad} X}(Y) \in \operatorname{LS}(\Gamma)$ for all $t \in \mathbb{R}$.

c) If $\pm X$ and $\pm Y$ belong to $\operatorname{LS}(\Gamma)$, then $\pm[X, Y] \in \operatorname{LS}(\Gamma)$.

d) $\operatorname{LS}(\Gamma)$ generates $L$ as a Lie algebra.

e) $\Gamma$ contains $\pm B$, where $B \in L \cap \eta, B$ is a fixed strongly regular element and the order on $\eta^{\prime}$ is relative to $B$.

f) If $\pm X \in \operatorname{LS}(\Gamma)$, then $\operatorname{LS}(\Gamma) \supset \oplus\{L(\alpha) \mid \alpha \in R, X(\alpha) \neq 0\}$.

g) For all $X \in L$, let $\varrho_{+}(X)=\max \{\operatorname{Re} \alpha(B) \mid \alpha \in R, \alpha>0, X(\alpha) \neq 0\}$ and $\varrho_{-}(X)=\min \{\operatorname{Re} \alpha(B) \mid \alpha \in R, \alpha<0, X(\alpha) \neq 0\}$, for $B \in \Gamma$ defined in e). Set $R(B)=\{\alpha(B) \mid \alpha \in R\}$.

If $X \in \operatorname{LS}(\Gamma)$ and if $\varrho_{+}(X) \notin R(B)$ (resp. $\varrho_{-}(X) \notin R(B)$ ) or if $\varrho_{+}(X)=$ $\varrho(B) \in R(B)$ (resp. $\varrho_{-}(X)=\varrho(B) \in R(B)$ ) and $\pm X(\varrho) \in \operatorname{LS}(\Gamma)$, then: $L(\alpha) \subset$ $\operatorname{LS}(\Gamma)$ for all $\alpha \in R$ such that $X(\alpha) \neq 0$ and $\operatorname{Re} \alpha(B)=\varrho_{+}(X)\left(\operatorname{resp} . \varrho_{-}(X)\right)$.

h) Let $r_{m}=\sup \left\{\left|\operatorname{Re} s_{i}(B)\right| \mid\left(S_{i}+\sigma_{L}\left(S_{i}\right)\right) \cap L \not \subset \operatorname{LS}(\Gamma)\right\}$, if $r_{m}=\varrho(B) \in$ $R(B)$, then $L(\varrho) \subset \operatorname{LS}(\Gamma)$ and $L(-\varrho) \subset \operatorname{LS}(\Gamma)$.

i) If $s \in \operatorname{Max}(R)$, there exists an $X_{+} \in \operatorname{LS}(\Gamma)$ such that $X_{+}(s) \neq 0$ and an $X_{-} \in \operatorname{LS}(\Gamma)$ such that $X_{-}(-s) \neq 0$.

Comments. Properties a) to i) are the only properties of $\operatorname{LS}(\Gamma)$ that we are going to use in the proof of Theorem 2. Note that they are not independent.

From the general classical theory of $[\mathrm{JK}]$, properties a), b), c) are always satisfied for the Lie saturated set $\operatorname{LS}(\Gamma)$ of any $\Gamma \subset L$. Property d) is the obvious classical necessary condition for controllability.

Property i) comes from the assumption (H3) of our theorem 2.

Property f) is an easy consequence of the property e), which states that $\Gamma$ contains a strongly regular element and its opposite (see proposition 11(d), page.173 of $[\mathrm{JK}]$ for a proof).

Property g) is also stated in proposition $11(\mathrm{a}, \mathrm{b}, \mathrm{c})$, page 173 of $[\mathrm{JK}]$. It is an easy consequence of the general properties of $\operatorname{LS}(\Gamma)$.

Property h) is, as in [JK], a consequence of assumption (H4) of our theorem 1 and of the fact that the Lie group $G$ has a finite center. Let us recall briefly what happens:

Consider $A^{*}=A_{0}+\sum\left\{A(\alpha)|| \operatorname{Re} \alpha(B) \mid \leq r_{m}\right\} . A^{*}$ is in $\operatorname{LS}(\Gamma)$ because $A \in$ $\operatorname{LS}(\Gamma), \operatorname{LS}(\Gamma)$ is convex and $L(\gamma) \subset \operatorname{LS}(\Gamma)$ for all $\gamma \in R$ such that $|\operatorname{Re} \gamma(B)|>r_{m}$. Assume that $r_{m}=\varrho(B) \in R(B)$. Then $\varrho \neq 0, \varrho \in R_{i}$ for some $i, 1 \leq i \leq k$. We claim that $S_{i}=\sigma_{L}\left(S_{i}\right): \sigma_{L}$ being an anti-automorphism of $L_{\mathbb{C}}, \sigma_{L}\left(S_{i}\right)$ is a simple ideal of $L_{\mathbb{C}}$. Since $\sigma_{L}\left(L_{\varrho}\right)=L_{\sigma(\varrho)}=L_{\varrho}$, so $\sigma_{L}\left(S_{i}\right) \cap S_{i} \supset L_{\varrho} \neq\{0\}$. Thus $\sigma_{L}\left(S_{i}\right)=S_{i}$. If $r_{m} \neq \operatorname{Re} s_{i}(B)$, then $\operatorname{Re} s_{i}(B)>r_{m}, S_{i} \cap L=\left(S_{i}+\sigma_{L}\left(S_{i}\right)\right) \cap L \subset$ $\mathrm{LS}(\Gamma)$. Additionally $L(\varrho), L(-\varrho) \subset \operatorname{LS}(\Gamma)$.

If $r_{m}=\operatorname{Re} s_{i}(B)$, by assumption $(\mathrm{H} 4)$, trace $(\operatorname{ad} A(\varrho) \circ$ ad $A(-\varrho))<0$, which implies that $Z=A(\varrho)+A(-\varrho)=A^{*}(\varrho)+A^{*}(-\varrho)$ is a compact vector field (i.e. 
the set $\{\exp (t Z) \mid t \in \mathbb{R}\}$ is relatively compact in $G$ ).

Since the projection $G \rightarrow \operatorname{Ad} G$ is a finite covering map, to show that $Z \in L$ is compact, it is sufficient to prove that it is compact in $\operatorname{Ad} G$. Let us recall some facts. Let $\tau: L_{\mathbb{C}} \rightarrow L_{\mathbb{C}}$ be the unique Weyl anti-involution commuting with $\sigma_{L}$. Then:

(i) $\tau\left(L_{\alpha}\right)=L_{-\alpha}$ for all $\alpha \in R$,

(ii) the hermitian form: $Z \in L_{\mathbb{C}} \rightarrow \operatorname{Kil}(Z, \tau(Z))$ is negative definite,

(iii) if $Z \in L_{\mathbb{C}}$ and $\tau(Z)=Z, Z$ is compact in $\operatorname{Ad}_{\mathbb{C}} G$, the adjoint group of $L_{\mathbb{C}}$.

Take any $X \in L(\varrho) \backslash\{0\} \subset L_{\varrho} \backslash\{0\}$, any $Y \in L(-\varrho) \backslash\{0\} \subset L_{-\varrho} \backslash\{0\}$ such that $\operatorname{Kil}(X, Y)<0$. Set $Z=X+Y, \tau(X) \in L_{-\varrho}$. So, $\tau(X)=c Y$ where $c \in \mathbb{C} \backslash\{0\}$. Since $\tau \circ \sigma_{L}=\sigma_{L} \circ \tau$ and $\sigma_{L}(X)=X$, we have $\tau(X)=\tau \circ \sigma_{L}(X)=$ $\sigma_{L} \circ \tau(X)=c^{*} Y$. So $c^{*}$, the conjugate of $c$, is equal to $c$, and $c$ is real. Also, by (ii) above, $0>\operatorname{Kil}(X, \tau(X))=c \operatorname{Kil}(X, Y)$. Hence, $c>0$.

If $c=1, \tau(Z)=Z$ and so $Z$ is compact.

If $c \neq 1$, let $\zeta=-1 / 2 \log c$ and $H=[X, Y]$, then $[H, X]=h X$ and $[H, Y]=$ $-h Y$ where $h \in \mathbb{R} \backslash\{0\}$. Let $Z_{1}=e^{(\zeta / h) \text { ad } H}(Z)$. Then it is easy to check that $\tau\left(Z_{1}\right)=Z_{1}$. Hence $Z_{1}$ is compact. Since $Z$ is conjugate to $Z_{1}$, it is also compact in $\operatorname{Ad} G$.

By proposition 11(b), page 173 of $[\mathrm{JK}]$ again, $A(\varrho) \in \operatorname{LS}(\Gamma)$ and $A(-\varrho) \in$ $\mathrm{LS}(\Gamma)$. Apply these considerations to $X=A(\varrho), Y=A(-\varrho), Z=X+Y$. Because $Z$ is compact, the closure of the semigroup $\{\exp (t Z) \mid t \geq 0\}$ contains the one-parameter group $\{\exp (t Z) \mid t \in \mathbb{R}\}$. Hence by the definition of $\operatorname{LS}(\Gamma)$, $\pm Z \in \operatorname{LS}(\Gamma)$. Property f) implies that $L(\varrho) \subset \operatorname{LS}(\Gamma), L(-\varrho) \subset \operatorname{LS}(\Gamma)$.

Hence the assumptions of our theorem 2 plus the general properties of $\operatorname{LS}(\Gamma)$ imply that for the "system" $\Gamma$ of theorem 2 , all the properties a) to i) above hold.

Therefore, theorem 2 follows from the following result.

THEOREM 3. If $\Gamma$ is a subset of $L$ satisfying conditions a) to i) above, then $\operatorname{LS}(\Gamma)=L$ and $\Gamma$ is transitive.

3. Sketch of the proof of the main result. In this section we show theorem 3 which, as explained in section 2, implies our main result.

Sketch of proof of theorem 3. Assume that $\operatorname{LS}(\Gamma) \neq L$. We define:

$$
\begin{aligned}
\nu & =\left\{i \mid 1 \leq i \leq k, \exists \alpha \in R_{i}, L(\alpha) \not \subset \operatorname{LS}(\Gamma)\right\} \\
& =\left\{i \mid\left(S_{i}+\sigma_{L}\left(S_{i}\right)\right) \cap L \not \subset \operatorname{LS}(\Gamma)\right\} .
\end{aligned}
$$

Let $r_{m}=\max \left\{\operatorname{Re} s_{i}(B) \mid i \in \nu\right\}$. Let $\mu=\left\{i \in \nu \mid \operatorname{Re}\left(s_{i}(B)\right)=r_{m}\right\}$. All the subalgebras

$$
\bigoplus_{i \in \mu} S_{i}, \quad \bigoplus_{i \in \nu} S_{i}, \quad \bigoplus_{i \notin \mu} S_{i}, \quad \bigoplus_{i \notin \nu} S_{i}
$$

are stable under $\sigma_{L}$. Let us denote by $\widehat{L}$ the semi-simple real subalgebra of $L$, $L \cap\left(\bigoplus_{i \in \mu} S_{i}\right)$. Then $\widehat{L}$ is not contained in $\operatorname{LS}(\Gamma)$ and $\widehat{L} \otimes_{\mathbb{R}} \mathbb{C}$ has the algebra 
$\widehat{\eta}=\bigoplus_{i \in \mu} \eta_{i}$ as a Cartan subalgebra. We have $\sigma_{L}(\widehat{\eta})=\widehat{\eta}$ and the set of roots of $\widehat{L} \otimes_{\mathbb{R}} \mathbb{C}$ with respect to $\widehat{\eta}$ is the set of restrictions to $\widehat{\eta}$ of elements of $\widehat{R}=$ $\bigcup_{i \in \mu} R_{i} \subset R$. $\operatorname{Max}(\widehat{R})$ for the order induced by $\eta^{\prime}$ is denoted by $M$. Let $\widehat{R}^{\prime}$ and $\widehat{R}^{\prime \prime}$ be the subsets defined in proposition 0 , relative to $\widehat{L}, \widehat{\eta}, \widehat{R}$ and the order induced on $(\widehat{\eta})^{\prime}=\operatorname{Hom}_{\mathbb{C}}(\widehat{\eta}, \mathbb{C})$ by $\eta^{\prime}$. The set $\widehat{R}$ is stable under $\sigma$ and by the map: $\alpha \rightarrow-\alpha$. The sets $\widehat{R}^{\prime}$ and $\widehat{R}^{\prime \prime}$ are stable under $\alpha \rightarrow-\alpha$.

Finally, $\widehat{R}^{\prime}=\bigcup_{i \in \mu} R_{i}^{\prime}$ and $\widehat{R}^{\prime \prime}=\bigcup_{i \in \mu} R_{i}^{\prime \prime}$.

For the proof of theorem 3, we need some lemmas.

Lemma 2. Assume that $\pm X \in \operatorname{LS}(\Gamma), Y \in \operatorname{LS}(\Gamma)$ and $X \neq 0$. If $\pm \operatorname{ad}^{2} X(Y) \in$ $\operatorname{LS}(\Gamma)$ then $L(\alpha) \subset \operatorname{LS}(\Gamma)$ for all $\alpha \in R$ such that $[X, Y](\alpha) \neq 0$.

The proof is elementary. It only makes use of the properties b), c), a), f) of LS $(\Gamma)$. (See $[$ EAGK]).

Lemma 3. i) Any of the following three assumptions below implies that $L(\alpha) \subset$ $\operatorname{LS}(\Gamma)$ :

1) $\alpha \in R$ and $|\operatorname{Re} \alpha(B)|>r_{m}$

2) $\alpha \in \pm M$,

3) $\alpha \in R,|\operatorname{Re} \alpha(B)|=r_{m}$ and there is an $Y \in \operatorname{LS}(\Gamma)$ such that $Y(\alpha) \neq 0$.

ii) If $\alpha \in \widehat{R}^{\prime}$ and if there exists an $Y \in \operatorname{LS}(\Gamma)$ such that $Y(\alpha) \neq 0$, then $L(\alpha) \subset \operatorname{LS}(\Gamma)$.

The proof uses lemma 2 and the properties (given in proposition 0 and corollary 0) of the sets $\widehat{R}, \widehat{R}^{\prime}, \widehat{R}^{\prime \prime}$ defined above. (See [EAGK] for details.)

LEMma 4. If $\alpha \in \pm M \cup \widehat{R}^{\prime}$ and $L(\alpha) \subset \operatorname{LS}(\Gamma)$ then, for $Y \in \operatorname{LS}(\Gamma), L(\alpha+\beta) \subset$ $\operatorname{LS}(\Gamma)$ for all $\beta \in R$ such that $Y(\beta) \neq 0$.

The proof of this lemma is very difficult and technical because the sets $M, \widehat{R}^{\prime}$ and $\widehat{R}^{\prime \prime}$ are not stable by $\sigma$ in general. In the proof all possible different cases are examined. Note that the proof is much simpler in the case where $L$ is a real normal form, since for every $\alpha \in R, \alpha=\sigma(\alpha)$, which implies that several difficult cases disappear (see [EAGK]).

Now, let $I$ be the subalgebra of $\widehat{L}$ generated by the $L(\alpha)$ such that $\alpha \in \pm M \cup \widehat{R}^{\prime}$ and $L(\alpha) \subset \operatorname{LS}(\Gamma)$. Assume that we have shown that $I$ is in fact an ideal of $\widehat{L}$. Then $I \otimes_{\mathbb{R}} \mathbb{C}$ is an ideal of $\widehat{L} \otimes_{\mathbb{R}} \mathbb{C}=\bigoplus_{i \in \mu} S_{i}$ and $I \otimes_{\mathbb{R}} \mathbb{C}=\bigoplus\left\{S_{i} \mid i \in \mu\right.$ and $\left.S_{i} \cap\left(I \otimes_{\mathbb{R}} \mathbb{C}\right) \neq\{0\}\right\}$. For all $i \in \mu$, by lemma $\left.3, \mathrm{i}\right), L\left(s_{i}\right) \subset I$ and so $\{0\} \neq L_{s_{i}} \subset S_{i} \cap\left(I \otimes_{\mathbb{R}} \mathbb{C}\right)$. Hence $I=\widehat{L}$. This yields a contradiction since $I \subset \operatorname{LS}(\Gamma)$ by its definition and the property c) of $\operatorname{LS}(\Gamma)$, but $\widehat{L}$ is not contained in $\operatorname{LS}(\Gamma)$.

To see that $I$ is an ideal of $\widehat{L}$, notice that $L$ is generated by the $X(\alpha)$ such that $X \in \operatorname{LS}(\Gamma)$, and $\alpha \in R$ (because $\Gamma$ contains $\pm B, B$ strongly regular). Also, $S_{i}$ is generated by the $X_{\alpha}$ such that $\alpha \in R_{i}$ and $X \in \operatorname{LS}(\Gamma)$. Hence, $\widehat{L}$ is generated by the $X(\alpha)$ such that $X \in \operatorname{LS}(\Gamma), X(\alpha) \neq 0, \alpha \in \widehat{R}$. Therefore $I$ is an ideal of 
$\widehat{L}$ provided that for any $\alpha \in \pm M \cup \widehat{R}^{\prime}$ such that $L(\alpha) \subset \operatorname{LS}(\Gamma)$, and any $\beta \in \widehat{R}$ such that there exists an $X \in \operatorname{LS}(\Gamma), X(\beta) \neq 0$ we have $[L(\alpha), X(\beta)] \subset I$.

If $\beta$ or $\sigma(\beta)$ belongs to $\pm M \cup \widehat{R}^{\prime}$, then lemma 3 , i), ii) shows that $L(\beta)=$ $L(\sigma(\beta)) \subset \operatorname{LS}(\Gamma)$, since $X(\beta)=X(\sigma(\beta)) \neq 0$. Hence $L(\beta) \subset I$ and since $I$ is an algebra, $[L(\alpha), L(\beta)] \subset I$.

Assume that $\beta$ and $\sigma(\beta) \in \widehat{R}^{\prime \prime}$. If $\alpha \in \pm M$ we see, by definition of $R^{\prime \prime}$, that neither $\alpha+\beta$ nor $\alpha+\sigma(\beta)$ is a root. Since $\widehat{R}^{\prime \prime}=-\widehat{R}^{\prime \prime}$ and $\pm M \cap \widehat{R} " \prime=\emptyset$ we deduce that $\alpha+\beta \neq 0$ and $\alpha+\sigma(\beta) \neq 0$. Then $[L(\alpha), L(\beta)]=\{0\} \subset I$. If $\alpha \in \widehat{R}^{\prime}$, then $\alpha+\beta \neq 0$ and $\alpha+\sigma(\beta) \neq 0$ because $\widehat{R}^{\prime}=-\widehat{R}^{\prime}$ and $\widehat{R}^{\prime} \cap \widehat{R} "=\emptyset$. Also, $[L(\alpha), L(\beta)] \neq\{0\}$ implies that at least one of the forms $\alpha+\beta, \alpha+\sigma(\beta)$ is a root. By proposition 0-2), if $\alpha+\beta \in R$ (resp. $\alpha+\sigma(\beta) \in R$ ) then $\alpha+\beta \in \widehat{R}^{\prime}$ (resp. $\left.\alpha+\sigma(\beta) \in \widehat{R}^{\prime}\right)$. Since $L(\alpha) \subset \operatorname{LS}(\Gamma), \alpha \in \widehat{R}^{\prime}, X(\sigma(\beta))=X(\beta) \neq 0$ and $X \in \operatorname{LS}(\Gamma)$, lemma 4 shows that $L(\alpha+\beta)+L(\alpha+\sigma(\beta)) \subset \operatorname{LS}(\Gamma)$. Hence, $L(\alpha+\beta)+L(\alpha+\sigma(\beta)) \subset I$. But $[L(\alpha), L(\beta)]=L(\alpha+\beta)+L(\alpha+\sigma(\beta))$ by lemma $0-\mathrm{f})$. This ends the proof of theorem 3.

\section{References}

[B] N. Bourbaki, Groupes et algèbres de Lie, Fasc. XXXVIII, chap. 7-8, Hermann, Paris, 1975.

[BJKS] B. Bonnard, V. Jurdjevic, I. Kupka and G. Sallet, Transitivity of families of invariant vector fields on the semi-direct products of Lie groups, Trans. Amer. Math. Soc. 271 (1982), 525-535.

[EA] R. El Assoudi, Accessibilité par des champs de vecteurs invariants à droite sur un groupe de Lie, Thèse de doctorat de l'Université Joseph Fourier, Grenoble, 1991.

[EAG] R. El Assoudi and J. P. Gauthier, Controllability of right invariant systems on real simple Lie groups of type $F_{4}, G_{2}, B_{n}$ and $C_{n}$, Math. Control Signals Systems 1 (1988), 293-301.

[EAGK] R. El Assoudi, J. P. Gauthier and I. Kupka, Controllability of right invariant systems on semi-simple Lie groups, Ann. Inst. Henri Poincaré, to appear.

[GB] J. P. Gauthier et G. Bornard, Controllabilité des systèmes bilinéaires, SIAM J. Control Optim. 20 (1982), 377-384.

[GKS] J. P. Gauthier, I. Ku pka and G. Sallet, Controllability of right invariant systems on real simple Lie groups, Systems Control Letters 5 (1984), 187-190.

[H] S. Helgason, Differential Geometry and Symmetric Spaces, Academic Press, New York, 1962.

[HHL] J. Hilgert, K. Hoffman and J. D. Lawson, Controllability of systems on a nilpotent Lie group, Beitr. Algebra Geom. 30 (1985), 185-190.

[HI] J. Hilgert, Max. semigroups and controllability in products of Lie groups, Arch. Math. (Basel) 49 (1987), 189-195.

[J] A. Joseph, The minimal orbit in a simple Lie algebra and its associated maximal ideal, Ann. Sci. Ecole Norm. Sup. (4) 9 (1976), 1-29.

[JK] V. Jurdjevic and I. Kupka, Controllability of right invariant systems on semisimple Lie groups and their homogeneous spaces, Ann. Inst. Fourier (Grenoble) 31 (4) (1981), 151-179. 
[L] J. D. Lawson, Maximal subsemigroups of Lie groups that are total, Proc. Edinburgh Math. Soc. 30 (1987), 479-501.

[LC] F. S. Leite and P. E. Crouch, Controllability on classical Lie groups, Math. Control Signals Systems 1, 1988, 31-42.

[W] G. Warner, Harmonic Analysis on Semi-simple Lie Groups 1, Springer, Berlin, 1972 . 